\title{
Tissue-specific differentially methylated regions of the human VASA gene are potentially associated with maturation arrest phenotype in the testis
}

\author{
Kazuhiro Sugimoto ${ }^{1}$, Eitetsu Koh ${ }^{1}$, Ho-Su $\operatorname{Sin}^{1}$, Yuji Maeda ${ }^{1}$, Kazutaka Narimoto ${ }^{1}$, Koji Izumi ${ }^{1}$, \\ Yoshitomo Kobori ${ }^{1}$, Eiko Kitamura ${ }^{2}$, Hiroki Nagase ${ }^{2}$, Atsumi Yoshida ${ }^{3}$ and Mikio Namiki ${ }^{1}$
}

Numerous CpG islands containing tissue-specific differentially methylated regions (TDMRs) are potential methylation sites in normal cells and tissues. The VASA (also known as DDX4) gene is believed to be under the control of TDMRs. A total of 131 male patients with idiopathic azoospermia or severe oligospermia were evaluated histologically, and the methylation status of CpG islands in the VASA gene was screened. Genome DNAs were obtained from testicular biopsy and modified with sodium bisulfite, and matrix-assisted laser desorption/ionization time-of-flight mass spectrometry (MALDI-TOF MS) was applied. This system is capable of analyzing both the methylated and unmethylated CpG island in the genome. The methylation analysis is conducted by an epigram as graphic data. On histological assessment, 17 of 131 patients revealed maturation arrest (MA). In all, 6 of the 17 patients showed particularly high VASA TDMR methylation rates, whereas the remaining 11 patients and controls had low methylation rates. This study may imply that the VASA TDMR methylation is significantly higher among patients with MA, in whom the VASA gene expression was silenced. This finding represents an important contribution to the molecular basis of meiotic arrest as one possible cause of idiopathic infertility.

Journal of Human Genetics (2009) 54, 450-456; doi:10.1038/jhg.2009.59; published online 24 July 2009

Keywords: CpG island; DDX4; MALDI-TOF MS; male infertility; spermatogenesis

\section{INTRODUCTION}

Spermatogenesis is a complex multistep process, mediated by different molecules, that encompasses the entire sequence of meiotic events from spermatogonia to mature spermatozoa in men. There is increasing evidence for genetic and epigenetic regulation of meiosis in the spermatogenesis of many model organisms. However, few attempts have been made at clinical investigation of this mechanism in humans. In particular, epigenetic changes are believed to influence male fertility. ${ }^{1,2}$ DNA methylation is an epigenetic modification that is related to changes in gene expression without any accompanying changes in the DNA sequence. ${ }^{3,4}$ The DNA methylation state at CpG dinucleotides, also known as CpG islands, is occasionally found in the promoter regions of many genes. CpG islands are generally not methylated during normal cell development, with the exception of imprinted genes and tissue-specific differentially methylated regions (TDMRs). Although each cell of an organism contains basically the same genome, gene activation is cell-specific as well as temporally and positionally mediated. TDMRs are hypomethylated in specific tissues and methylated in all other tissues, ${ }^{5,6}$ in which DNA methylation is involved in gene silencing. As a result, genes with hypomethylated TDMRs are expressed in a tissue-specific manner.
Several studies have shown that TDMRs are located upstream of the regulatory regions in the mouse. ${ }^{7,8}$ In general, repeat sequences, promoters of tissue-specific genes and gene-coding regions are hypermethylated. However, numerous $\mathrm{CpG}$ islands containing TDMRs are potential methylation sites in normal cells and tissues. A genome-wide epigenetic analysis recently showed the evolutionary conservation of tissue-specific methylation in human brain cells, keratinocytes and peripheral blood lymphocytes. ${ }^{9,10}$

Furthermore, the VASA (also known as DDX4) gene is believed to be under the control of TDMRs. Kitamura et al. ${ }^{11}$ found unmethylated and methylated $\mathrm{CpG}$ islands in the human testis, as bisulfite sequencing results showed $50 \%$ of clones hypomethylated and $50 \%$ of clones hypermethylated. VASA encodes a member of the DEAD (Asp-Glu-Ala-Asp) box family of adenosine triphosphate (ATP)dependent RNA helicases. This indicates that the methylation status of the VASA promoter CpG islands with TDMs was associated with spermatogenesis and the regulation of VASA gene expression.

The VASA ortholog in Drosophila vasa, is required for spermatogenesis and oogenesis by a mechanism that involves regulating the translation of mRNAs that are essential for differentiation. ${ }^{12}$ The Mouse Vasa Homolog $(\mathrm{Mvh})$ gene is expressed specifically in the

${ }^{1}$ Department of Integrative Cancer Therapy and Urology, Kanazawa University Graduate School of Medical Science, Kanazawa, Japan; ${ }^{2}$ Department of Advanced Medical Science, Division of Cancer Genetics, Nihon University School of Medicine, Tokyo, Japan and ${ }^{3}$ Reproduction Center Kiba Park Clinic, Tokyo, Japan

Correspondence: Dr E Koh, Department of Integrative Cancer Therapy and Urology, Kanazawa University Graduate School of Medical Science, 13-1 Takara-machi, Kanazawa, Ishikawa 920-8141, Japan.

E-mail: kohei@med.kanazawa-u.ac.jp

Received 20 April 2009; revised 8 June 2009; accepted 13 June 2009; published online 24 July 2009 
developing germ cell lineage during embryogenesis. ${ }^{13}$ It has been reported that male mice that are homozygous for a targeted mutation of $M v h$ show a reproductive deficiency in which they produce no spermatozoa in the testes. Instead, premeiotic germ cells cease differentiation by the zygotene stage and undergo apoptotic death. ${ }^{14}$ In humans, the gene is mapped to the human chromosome 5q; its expression is restricted to the testes in males and is undetectable in somatic tissues. ${ }^{15}$

Moreover, the VASA gene modulates the RNA structure, which is a crucial step in many fundamental biological processes. A variety of RNA metabolic processes, including transcription, ribosomal biogenesis, RNA splicing, editing, transport and translational events, are also controlled by VASA. ${ }^{16-21}$ In addition, recent studies have shown VASA gene involvement in RNA metabolism and micro RNA processing. ${ }^{22}$

The aim of this study was to test the possibility that the DNA methylation states in the VASA promoter CpG islands may contribute to some forms of human idiopathic male infertility. In this study, we also applied the matrix-assisted laser desorption/ionization time-offlight mass spectrometry (MALDI-TOF MS) analysis to identify DNA methylation states using testicular genome DNA.

\section{MATERIALS AND METHODS}

\section{Patients}

We recruited 131 patients with azoospermia or severe oligozoospermia (sperm concentration $<1 \times 10^{6}$ per $\mathrm{ml}$ ), excluding abnormal karyotypes and Y chromosome microdeletion. ${ }^{23}$ All 131 patients underwent either open testicular biopsy or retrieval of sperm from testicular tissues to evaluate histology while attempting to extract testicular sperm. The Ethics Committee of the Kanazawa University Hospital approved the study, and informed consent was obtained from all participants.

\section{Histological evaluation of testicular biopsies}

Testicular specimens were fixed in Bouin's solution, and paraffin slides were prepared and stained by hematoxylin and eosin staining for histological evaluation. To diagnose the spermatogenic pattern, samples were obtained from at least three different (lower, middle and upper) areas in the testis. Diagnostic biopsies included the evaluation of more than 100 sections per biopsy of seminiferous tubules per biopsy. ${ }^{24,25}$ Testicular histology was classified into hypospermatogenesis (reduction in the degree of normal spermatogenic cells), maturation arrest (MA) (lack of late stage of spermatogenesis), Sertoli cell-only syndrome(lack of germ cells in seminiferous tubules) and tubular sclerosis (no germ cell or Sertoli cell present in the seminiferous tube). ${ }^{26}$

\section{Isolation of total RNA and genomic DNA}

All of the testicular tissue specimens obtained from biopsy were used for the isolation of total RNA and testicular genomic DNA. The TRIzol Reagent (Invitrogen, Carlsbad, CA, USA) was available for extracting both molecules from the same specimen. Total RNA was treated using the TURBO DNA-free kit (Ambion, Austin, TX, USA) for removing any residual genomic DNA. Single-stranded cDNA was synthesized using a High-Capacity cDNA Archive Kit (Applied Biosystems, Foster City, CA, USA).

\section{RT-PCR}

To define the testis-specific expression of the VASA gene, reverse transcriptase (RT)-PCR was carried out on cDNA samples obtained from normal human tissues, including the testis, muscle, kidney, heart, brain and colon tissues, provided by Professor Nagai (Nihon University, Tokyo, Japan). Taq DNA polymerase was used for PCR reactions (TaKaRa Bio, Shiga, Japan). PCR amplifications for both VASA and glyceraldehyde-3-phosphate dehydrogenase $(G A P D H)$ were carried out with the following parameters: $95^{\circ} \mathrm{C}$ for $5 \mathrm{~min} ; 29$ cycles of denaturing at $95^{\circ} \mathrm{C}$ for $30 \mathrm{~s}$, annealing at $60^{\circ} \mathrm{C}$ for $30 \mathrm{~s}$, extension at $72{ }^{\circ} \mathrm{C}$ for $30 \mathrm{~s}$ and a final incubation at $72^{\circ} \mathrm{C}$ for $10 \mathrm{~min}$. The PCR products were resolved on $1.2 \%$ agarose gels. The specific primer pairs used for VASA (NM_024415) and GAPDH (NM_002046) amplification were 5'-CAAGAG AGGCGGCTATCGAGAT- $3^{\prime}$ and $5^{\prime}$-GTCAGAATTGCTGGTGGTGCATC- $3^{\prime}$ (483 bp) for VASA and 5'-GACCACAGTCCATGCCATCA-3' and 5'-TCCACCA CCCTGTTGCTGTA-3' (453 bp) for GAPDH.

\section{Confirmation of TDMR}

Kitamura et al. ${ }^{11}$ showed that the presence of TDMRs that are located upstream of the VASA gene is associated with the testis-specific expression. The region is -1127 to -760 ( $368 \mathrm{bp}$ amplicon) from the transcription start site, and contains a promoter $\mathrm{CpG}$ island with $30 \mathrm{CpG}$ binucleotides, a $\mathrm{G}+\mathrm{C}$ content of $63.5 \%$ and an observed/expected CpG ratio of 1.01 (http://genome.ucsc.edu/ index.html?org $=$ Human\&db=hg18\&hgsid $=107086429)$. We analyzed this amplicon using tissue samples obtained from patients.

\section{Bisulfite treatment}

Isolated genomic DNA $(1 \mu \mathrm{g})$ obtained from testicular specimens was converted with sodium bisulfite using the EZ DNA methylation kit (Zymo Research, Orange, CA, USA) according to the manufacturer's instructions.

\section{PCR and in vitro transcription}

The position of the VASA promoter CpG island and the amplicon analyzed in this study are shown in Figure 1. Bisulfite-treated DNA was subjected to PCR with the specific primer pair. A reverse primer of 5'-CAGTAATACGACTCACT ATAGGGAGAAGGCTCATCCACACTTTAACCAAAAATC- $3^{\prime}$ was tagged with a T7 promoter sequence. The forward primer used was $5^{\prime}$-AGGAAGAGA GGTTTTGTATTTATAGGTTTAATAGGTTATT-3'. Amplification of $1 \mu \mathrm{l}$ of bisulfite-treated DNA $\left(\sim 20 \mathrm{ng} \mu \mathrm{l}^{-1}\right)$ was carried out using HotStar Taq Polymerase (Qiagen, Valencia, CA, USA) in $5 \mu \mathrm{l}$ of reaction volume, using PCR primers at a final concentration of $200 \mu \mathrm{M}$. PCR amplification was carried out with the following parameters: $94^{\circ} \mathrm{C}$ for $15 \mathrm{~min}$ hot start, followed by 45 cycles of denaturing at $94^{\circ} \mathrm{C}$ for $20 \mathrm{~s}$, annealing at $58^{\circ} \mathrm{C}$ for $45 \mathrm{~s}$, extension at $72^{\circ} \mathrm{C}$ for $1 \mathrm{~min}$ and a final incubation at $72^{\circ} \mathrm{C}$ for $3 \mathrm{~min}$. After shrimp alkaline phosphatase treatment, $2 \mu \mathrm{l}$ of the PCR products were used as a template for in vitro transcription to obtain RNA transcripts. The reverse product was subjected to base-specific cleavage, and the resulting samples were conditioned and spotted on a 384-pad SpectroCHIP (Sequenom, San Diego, CA, USA) using a MassARRAY nanodispenser (Samsung, Irvine, CA, USA), followed by spectral acquisition on a MassARRAY Analyzer Compact MALDI-TOF MS (Sequenom).

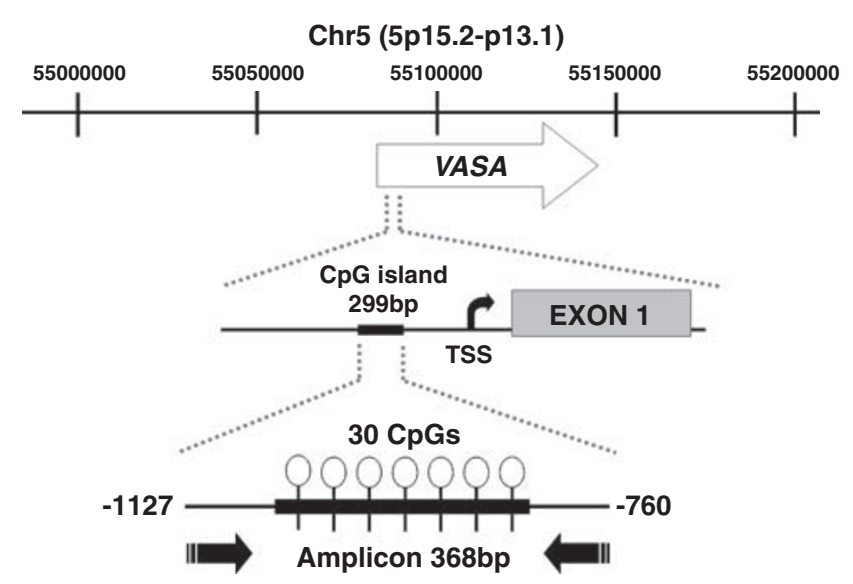

Figure 1 Chromosomal location of VASA. This map is based on the March 2006 human reference sequence (NCBI Build 36.1). VASA is shown as an arrow, with an arrowhead indicating the orientation of the gene. CpG islands are depicted with the number of CpGs. The amplicon is designed from -1127 to -760 (368 bp) from the transcription start site (TSS). 
Table 1 Clinical characteristics of MA patients with high methylation

\begin{tabular}{|c|c|c|c|c|c|c|c|c|c|c|c|}
\hline & $\begin{array}{c}\text { Age } \\
\text { (years) }\end{array}$ & $\begin{array}{c}\text { Semen } \\
\text { (sperm counts) }\end{array}$ & $\begin{array}{c}F S H \\
\left(m / \cup \mathrm{ml}^{-1}\right)\end{array}$ & $\begin{array}{c}L H \\
\left(m / \cup m l^{-1}\right)\end{array}$ & $\begin{array}{c}\text { Testosterone } \\
\left(\text { ng } \mathrm{ml}^{-1}\right)\end{array}$ & TESE & $\begin{array}{l}\text { Sperm } \\
\text { retrieval }\end{array}$ & ICSI & Fertilization & Pregnancy & Childbirth \\
\hline MA1 & 31 & 0 & 13.8 & 1.9 & 3.6 & Yes & Yes & Yes & Yes & Yes & Yes \\
\hline MA2 & 26 & 0 & 36.4 & 9.1 & 7.1 & Yes & No & $N / P$ & - & - & - \\
\hline MA3 & 44 & 0 & 25.7 & 7.3 & 2.9 & Yes & Yes & Yes & Yes & No & No \\
\hline MA4 & 33 & A few ${ }^{a}$ & 8.5 & 1.6 & 5.8 & Yes & Yes & Yes & $N / P$ & - & - \\
\hline MA5 & 34 & 0 & 12.4 & 2.8 & 5.8 & Yes & Yes & Yes & Yes & Yes & Yes \\
\hline MA6 & 35 & 0 & 43.33 & 19.83 & 2.78 & Yes & Yes & Yes & Yes & Yes & Yes \\
\hline
\end{tabular}

Abbreviations: ICSI, intra-cytospermic sperm injection; MA, maturation arrest; N/P not performed; TESE, testicular sperm extraction including microdissection. ${ }^{a}$ After centrifugation.

\section{MALDI-TOF MS analysis}

Matrix-assisted laser desorption/ionization time-of-flight mass spectrometry was used for the high-throughput quantitative DNA methylation assay. ${ }^{27}$ This system analyzed base-specific cleaved amplification products. In this study, we used the MassARRAY Compact system (Sequenom). The resultant methylation calls were conducted using EpiTyper software v1.0 (Sequenom) for generating quantitative results per cleavage fragment (known as $\mathrm{CpG}$ unit), including each CpG site or the aggregate of multiple CpG sites. The graphic data obtained from methylation analysis using MassARRAY were expressed as an epigram.

\section{Real-time quantitative RT-PCR}

For expression analysis of the human VASA gene, we used real-time quantitative RT-PCR. The generated sample cDNA was amplified on a LightCycler system (Roche Applied Science, Basel, Switzerland) using a LightCycler TaqMan Master (Roche Diagnostics, Mannheim, Germany). The TaqMan probe was purchased from Roche Diagnostics. Universal probes (nos 62 and 60) were used for VASA and GAPDH, respectively. Each universal probe was designed according to the Universal Probe Assay Design Center (https://www.rocheapplied-science.com/sis/rtpcr/upl/index.jsp). An additional primer pair used for VASA expression was 5'-GGAGTTGGAAAGAGTTTTGGAA-3' and 5'-CAGAAACCAGAGCTATCACCATC-3' (74bp). The primer pair used for GAPDH expression was 5'-AGCCACATCGCTCAGACA- $3^{\prime}$ and 5'-GCCCAATA CGACCAAATCC-3' (66 bp). To normalize the VASA mRNA expression with respect to sample-to-sample differences in RNA input, RNA quality and reverse transcriptase efficiency, we amplified the housekeeping gene, GAPDH. We obtained the copy numbers of VASA and GAPDH according to the respective standard curves. The results were determined by the ratio of $V A S A-G A P D H$ in each cDNA sample. All PCR reactions were carried out in a total volume of $20 \mu \mathrm{l}$ containing $4 \mu \mathrm{l}$ of $5 \times$ LightCycler TaqMan Master (Roche Diagnostics, final concentration: $1 \times$ ), $0.3 \mu$ of $10 \mu \mathrm{moll}^{-1}$ TaqMan probe (Roche Diagnostics, final concentration: $150 \mu \mathrm{moll}^{-1}$ ), $1 \mu \mathrm{l}$ of $10 \mu \mathrm{mol} \mathrm{l}^{-1}$ of each primer (final concentration: $500 \mu \mathrm{moll} \mathrm{l}^{-1}$ ), $2 \mu \mathrm{l}$ of sample cDNA, and $11.6 \mu \mathrm{l}$ of dietyl pyrocarbonate (DEPC)-treated water. Water was used in the negative controls instead of the cDNA template. Amplifications of $V A S A$ and GAPDH were performed in triplicate for each sample. The thermal cycling conditions were $10 \mathrm{~min}$ at $95^{\circ} \mathrm{C}$, followed by 50 cycles at $95^{\circ} \mathrm{C}$ for $10 \mathrm{~s}$ and 50 cycles at $60^{\circ} \mathrm{C}$ for $20 \mathrm{~s}$ for both VASA and GAPDH. The number of $V A S A$ and GAPDH transcripts in each sample was calculated with the LightCycler software, using these standard curves.

\section{Sample collection}

Several normal human tissue samples were obtained by organ donation from autopsy cases at the Pathology Division of the Nihon University School of Medicine in Tokyo, Japan. This study was approved by the Ethics Committees of both the Kanazawa University Graduate School of Medical Science and the Nihon University School of Medicine. Informed consent was obtained from all study participants. In cases for whom tissues were received through organ donation, bereaved families or relatives provided informed, written consent.

\section{Statistical analysis}

Data were analyzed using the Statistical Package for the Social Sciences statistical software version 11.0 (SPSS, Chicago, IL, USA). Mann-Whitney's $U$-test was used to compare the average methylation rate between patients within the MA and control groups. Welch's $t$-test was used to compare VASA mRNA expression between two groups of MA . Student's $t$-test was used to examine the correlation between hormone levels. Differences were considered as statistically significant at $P<0.05$.

\section{RESULTS}

\section{Characterization of patients by histological evaluation}

On histological assessment, spermatogenesis was classified as normal in 39 patients. In the group with normal spermatogenesis, 26 patients had obstructive azoospermia, 6 had retrograde ejaculation and 7 patients presented with unknown causes. We defined these patients as the control group. An additional 25 patients were diagnosed with hypospermatogenesis. These hypospermatogenetic patients were excluded from this study, because testicular biopsy revealed the presence of late spermatogenesis and a few spermatozoa.

Histology also revealed MA in testicular biopsy results obtained from 17 patients, who were defined as the MA group in this study. Although all MA patients lacked late spermatogenesis when examined histologically, an examination of ejaculate revealed that some had few spermatozoa in the ejaculate (MA4, 8, 27), as shown in Tables 1 and 2. The mean ages of the control and MA groups ( \pm s.d.) were $36.3 \pm 7.1$ and $35.5 \pm 5.9$ years, respectively. In addition, the remaining 50 patients had either a Sertoli cell-only phenotype or tubular sclerosis identified by histological analysis and were also excluded from this study because of the absence of germ cells.

\section{VASA gene expression}

The expression of VASA was detected by RT-PCR in human testicular tissues. No VASA expression was detected in the other five tissue types (Figure 2). Thus, the VASA gene was specifically expressed in the testis tissue.

\section{Confirmation of TDMR in normal tissues}

After bisulphate treatment, PCR was performed. These amplicons were screened using MassARRAY to evaluate the methylation status of VASA promoter CpG islands in different normal tissues.

We screened normal tissues, including the testis; the graphic data illustrated the methylation status within each amplicon (Figure 3). VASA promoter CpG islands were specifically hypomethylated in the testis, whereas five other tissue types were highly methylated. 
Table 2 Clinical characteristics of MA patients with low methylation

\begin{tabular}{|c|c|c|c|c|c|c|c|c|c|c|c|}
\hline & Age (years) & $\begin{array}{c}\text { Semen } \\
\text { (sperm counts) }\end{array}$ & $\begin{array}{c}F S H \\
\left(m / \cup m^{-1}\right)\end{array}$ & $\begin{array}{c}L H \\
\left(m / \cup m I^{-1}\right)\end{array}$ & $\begin{array}{c}\text { Testosterone } \\
\left(n g \mathrm{ml}^{-1}\right)\end{array}$ & TESE & $\begin{array}{l}\text { Sperm } \\
\text { retrieval }\end{array}$ & $|C S|$ & Fertilization & Pregnancy & Childbirth \\
\hline MA7 & 35 & 0 & 3.63 & 1.06 & 4.99 & Yes & Yes & Yes & Yes & Yes & Yes \\
\hline MA8 & 39 & $0.9 \times 106$ & 6.17 & 4.4 & 4.02 & $\mathrm{~N} / \mathrm{P}$ & Yes & Yes & Yes & Yes & Yes \\
\hline MA9 & 37 & 0 & 2.99 & 2.78 & 5.77 & Yes & Yes & Yes & Yes & Yes & Yes \\
\hline MA10 & 32 & 0 & 5.69 & 4.22 & 6.51 & Yes & Yes & Yes & Yes & Yes & Yes \\
\hline MA11 & 32 & 0 & 2.14 & 1.15 & 4.09 & Yes & No & $\mathrm{N} / \mathrm{P}$ & - & - & - \\
\hline MA12 & 27 & 0 & 23.7 & 11.0 & 4.3 & Yes & Yes & Yes & Yes & Yes & Yes \\
\hline MA13 & 42 & 0 & 7.66 & 2.06 & 4.6 & Yes & No & $N / P$ & - & - & - \\
\hline MA14 & 48 & 0 & 3.34 & 1.85 & 5.93 & Yes & Yes & Yes & Yes & Yes & Yes \\
\hline MA15 & 27 & A few ${ }^{a}$ & $N / A$ & $N / A$ & $N / A$ & Yes & Yes & Yes & Yes & Yes & Yes \\
\hline MA16 & 31 & 0 & 2.8 & 1 & 4.11 & Yes & Yes & Yes & Yes & No & - \\
\hline MA17 & 41 & 0 & 3.4 & 3.29 & 8.15 & Yes & Yes & Yes & Yes & Yes & Yes \\
\hline
\end{tabular}

Abbreviations: ICSI, intracytospermic sperm injection; MA, maturation arrest; N/A, not available; N/P, not performed; TESE; testicular sperm extraction including microdissection. aAfter centrifugation.

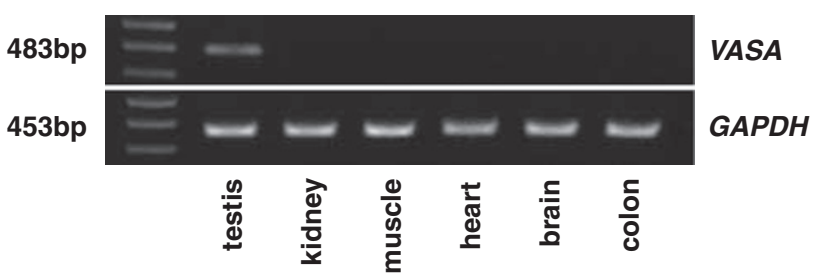

Figure 2 Tissue-specific expression of VASA in normal human tissues. cDNAs were prepared from the testis, kidney, muscle, heart, brain and pancreas. GAPDH was used as a positive control.

The evaluation of VASA TDMR methylation state in the testicular genome

We analyzed VASA gene TDMRs (that is, VASA promoter CpG islands) using MassARRAY. All samples were evaluated using testicular genome DNA. All controls $(n=39)$ are hypomethylated in the TDMR of the VASA gene; the average rate is $16.3 \pm 4.83 \%$ (Figure 4 ). In six patients of the MA group ( $n=17)$, the methylation rate of each CpG unit within an amplicon is especially high; the average rate is $77.4 \pm 3.07 \%$. The methylation rate was low in the remaining 11 patients, with an average of $29.3 \pm 2.68 \%$ (Figure 5). The methylation rate of the former was significantly higher than that of the latter $(P<0.0001)$. It has been suggested that some MAs might account for the methylated TDMRs in this study.

In 25 patients of the hypospermatogenetic group, the variability of the methylation rate is great, and the average rate is $45 \pm 21.4 \%$. All of the remaining 50 samples of VASA TDMRs are methylated; the average methylation rate of patients with Sertoli cell-only and tubular sclerosis was $88.0 \pm 8.7 \%$.

\section{Expression study of methylated and unmethylated TDMRs} in the MA group

To examine the expression of the VASA gene, VASA mRNA was measured using real-time quantitative RT-PCR. The average methylation rate and VASA gene expression levels are depicted as a scatter plot in Figure 6a. The expression level of VASA in the methylated group (mean \pm s.e.m.: $0.00132 \pm 0.00033$ ) was significantly lower than in the remaining 11 patients $(0.01391 \pm 0.00428, P=0.0150)$, Figure $6 b$. These results show that highly methylated TDMRs result in low levels of gene expression. Thus, the alteration of methylation within the VASA promoter may greatly affect expression, resulting in meiotic failure.

\section{Clinical characteristics of patients with methylated} and unmethylated TDMRs

The general characteristics and clinical parameters of patients with the MA phenotype are summarized in Table 1. Methylation state is classified into two groups, namely high methylation (HM) and low methylation (LM) groups as shown in Figures $6 \mathrm{a}$ and b. In the HM group $(n=6)$, all patients but one (MA4) had azoospermia in the ejaculate. In the LM group $(n=11)$, all patients except two (MA8, MA15) had azoospermia in the ejaculate. The levels of folliclestimulating hormone (FSH), luteinizing hormone ( $\mathrm{LH})$ and testosterone $(\mathrm{T})$ in the $\mathrm{HM}$ group were $23.4 \pm 5.5,7.1 \pm 2.8$ and $4.6 \pm 0.7$, respectively. By contrast, the levels of FSH, LH and T in the LH group were $6.1 \pm 2.1,3.3 \pm 0.9$ and $5.2 \pm 0.4$, respectively. The FSH level in the HM group was significantly higher compared with that in the LM group $(P=0.037)$. There was no significant difference in levels of $\mathrm{LH}$ $(P=0.25)$ or $\mathrm{T}(P=0.52)$ between the HM and LM groups. This finding leads to the suggestion that the HM rates in the VASA TDMR may be more harmful to spermatogenesis than the LM rates.

\section{DISCUSSION}

In this study, we identified patients with MA who had a relative HM status. A reduction in VASA expression because of its promoter CpG island methylation is potentially responsible for the MA phenotype.

It is well known that the human VASA expression is germ cellspecific. To evaluate the methylation status of patients' DNA, we should collect a pure population of germ cells; however, this is extremely difficult because of the heterogenic appearance of clinical samples. Therefore, we applied the MALDI-TOF MS system, which evaluated the rate of methylation levels in the total cell population mixture, but not in individually cloned DNA methylation.

In this study, we confirmed the moderate spermatocyte count in all 17 samples by histological diagnosis. To characterize the effect of methylation rate and the expression of VASA in MA patients, a total of 17 testicular biopsy samples were examined; 6 patients had a relatively high VASA expression and also indicated a significantly lower VASA expression compared with the remaining $11 \mathrm{MA}$ patients. Needless to say, the genomic DNA extracted from these testicular biopsies may not represent the methylation level in only germ cells. These testicular biopsies contain many somatic cells, such as Leydig cells, Sertoli cells, 


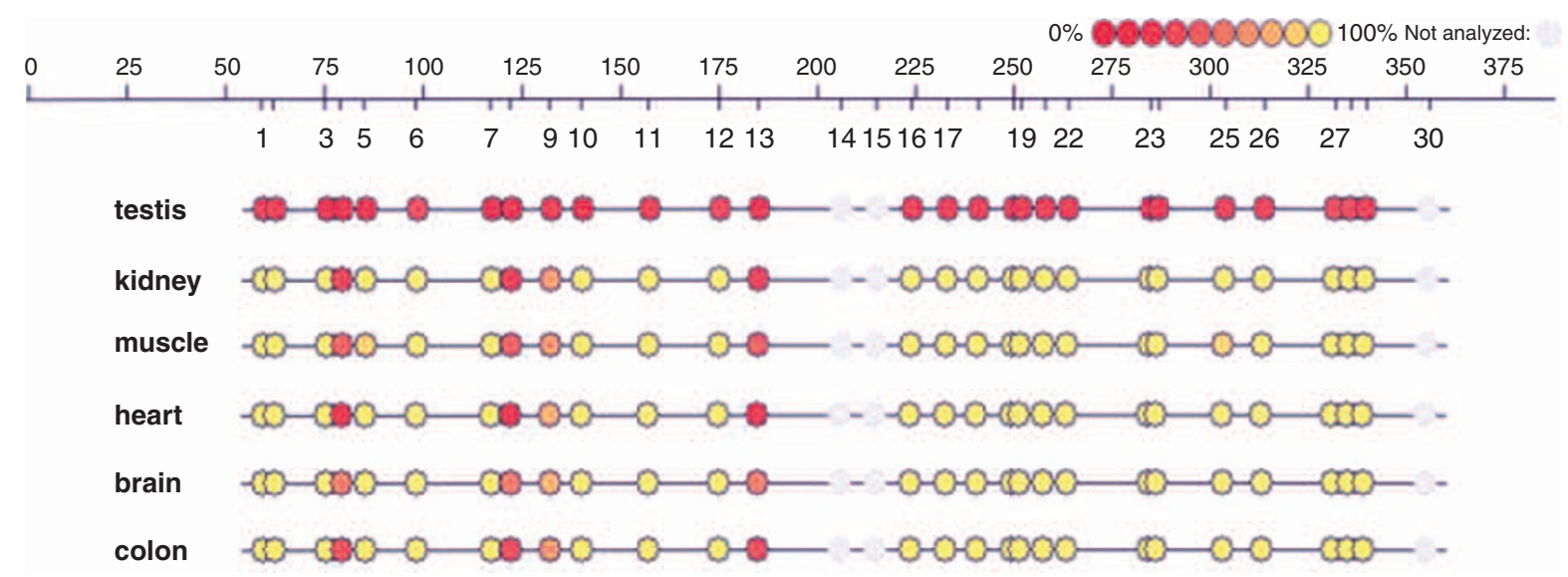

Figure 3 Epigram of the VASA tissue-specific differentially methylated region (TDMR) methylation state in normal human tissues. The upper ruler indicates the location of amplicon (368 bp), and the lower scale indicates the CpG site. Circles mark the positions of CpG sites, and dashed lines annotate CpG sites that could not be analyzed. The color scale in each top right corner indicates the methylation level. Red circles indicate that the TDMR is unmethylated. Gradation of circles from red to yellow indicates the rates of methylation ranging from 0 to $100 \%$. The epigram is shown for six normal human tissues (testis, kidney, muscle, heart, brain and colon).

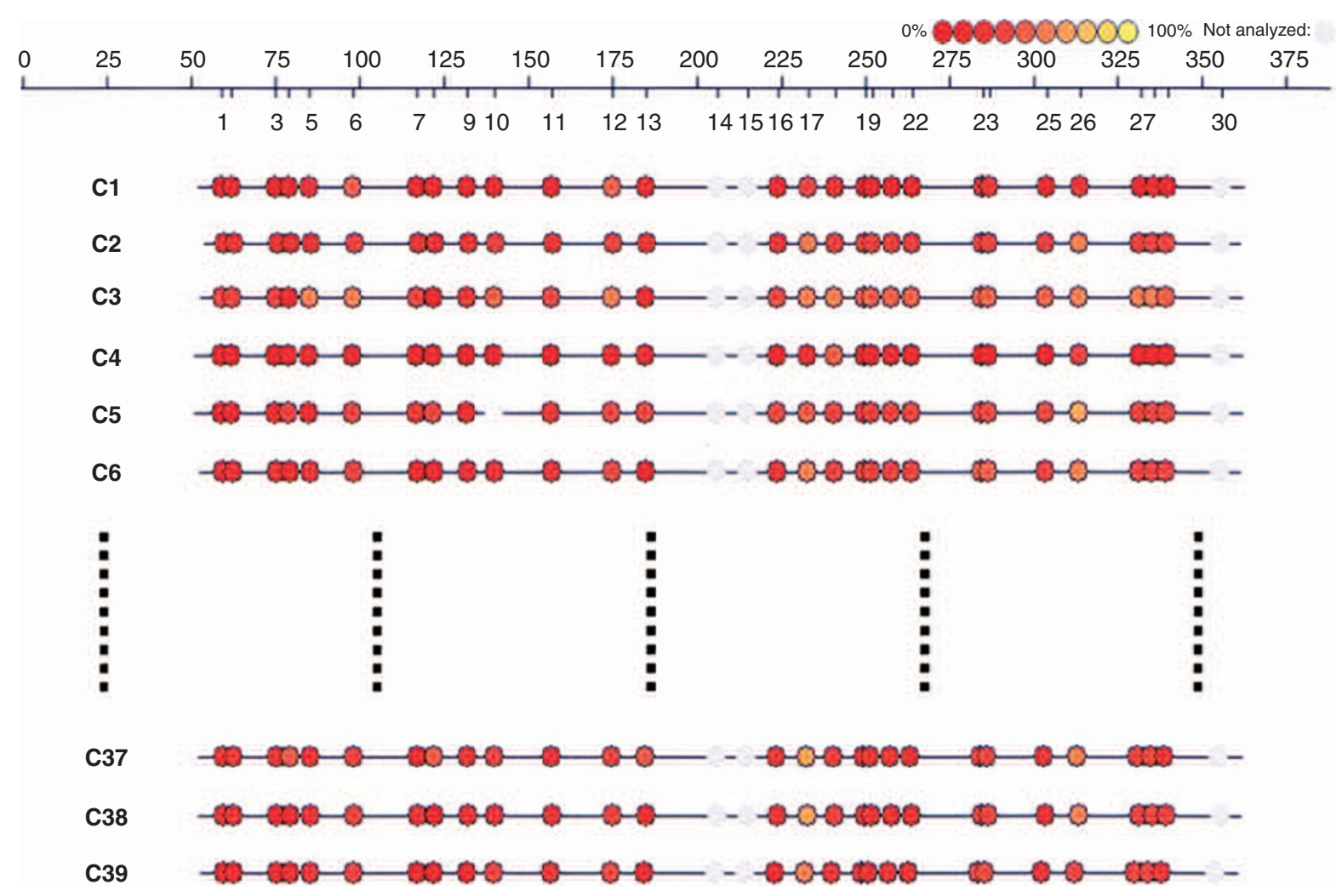

Figure 4 Epigram of the VASA tissue-specific differentially methylated region (TDMR) methylation state in control patients ( $n=39$ ). The upper ruler indicates the location of amplicon (368 bp), and the lower scale indicates the CpG site. Circles mark the positions of CpG sites, and dashed lines annotate CpG sites that could not be analyzed. The color scale in each top right corner indicates the methylation level. Red circles indicate that the TDMR is unmethylated. The gradation of circles from red to yellow indicates the rates of methylation ranging from 0 to $100 \%$.

etc. In the extreme case of Sertoli cell-only or tubular sclerosis in the remaining 50 patients, the VASA TDMR methylation rate indicated a high value as expected. These observations are consistent with the results obtained by Kitamura et al. ${ }^{11}$ Therefore, an aberrant methylation of VASA led to the molecular basis of meiotic arrest as one possible cause of idiopathic infertility.
In animal experiments, Tanaka et al. generated a mutation in the mouse Vasa homolog gene $(M v h)$ and showed that male knockout mice for $M v h$ produced no spermatozoa in the testes. Histological findings revealed that spermatogenesis was arrested in $M v h$-deficient mice early in meiosis. ${ }^{14}$ Interestingly, these histological findings were consistent with those observed in our six patients with histologically 

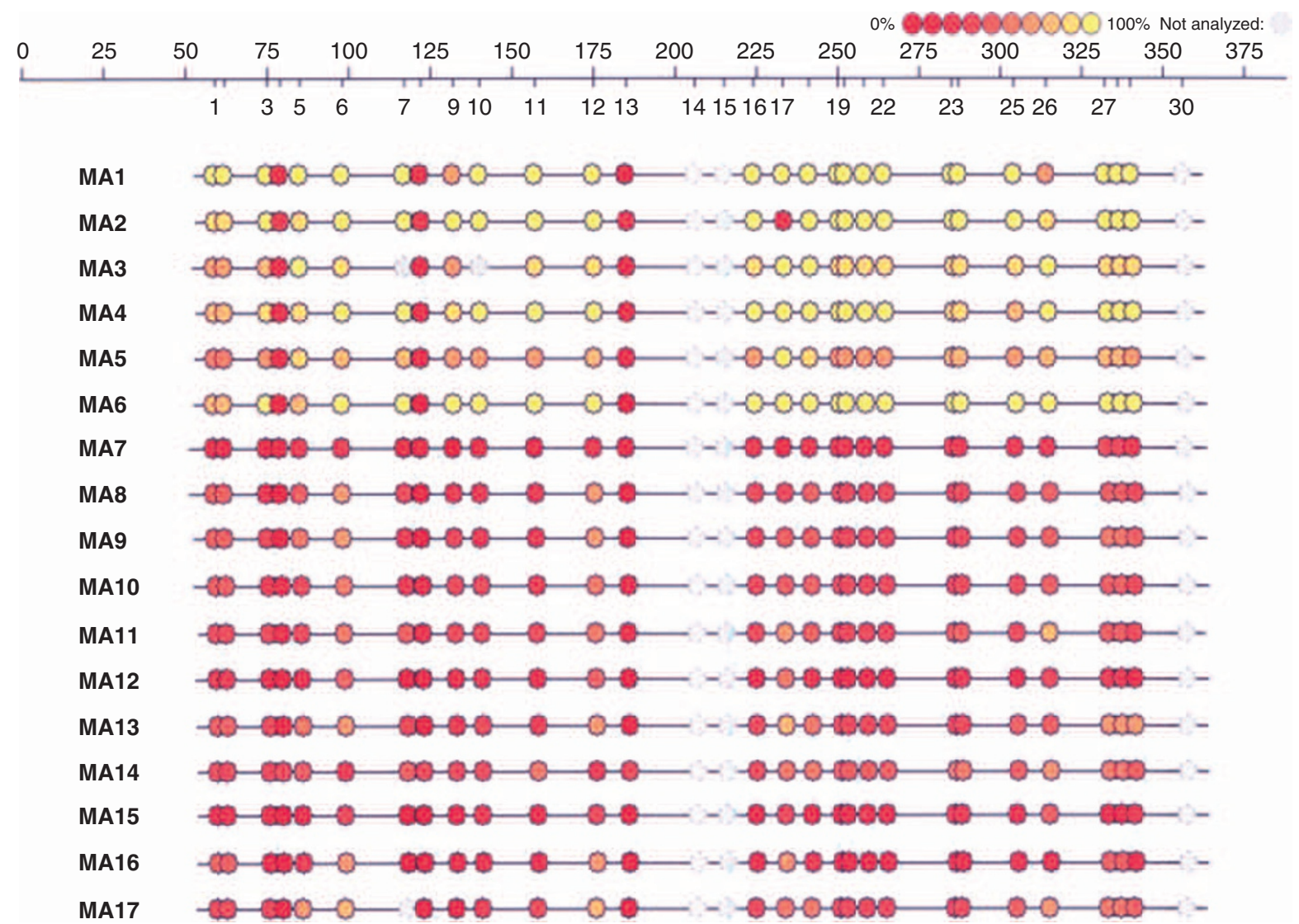

Figure 5 Epigram of methylation status in tissue-specific differentially methylated region (TDMR) of VASA in 17 patients with maturation arrest. The upper ruler indicates the location of amplicon (368 bp), and the lower scale indicates the CpG site. Circles mark the positions of $\mathrm{CpG}$ sites, and dashed lines annotate $\mathrm{CpG}$ sites that could not be analyzed. The color scale in each top right corner indicates the methylation level. Red circles indicate that the TDMR is unmethylated. Gradation of circles from red to yellow indicates the rates of methylation ranging from 0 to $100 \%$.
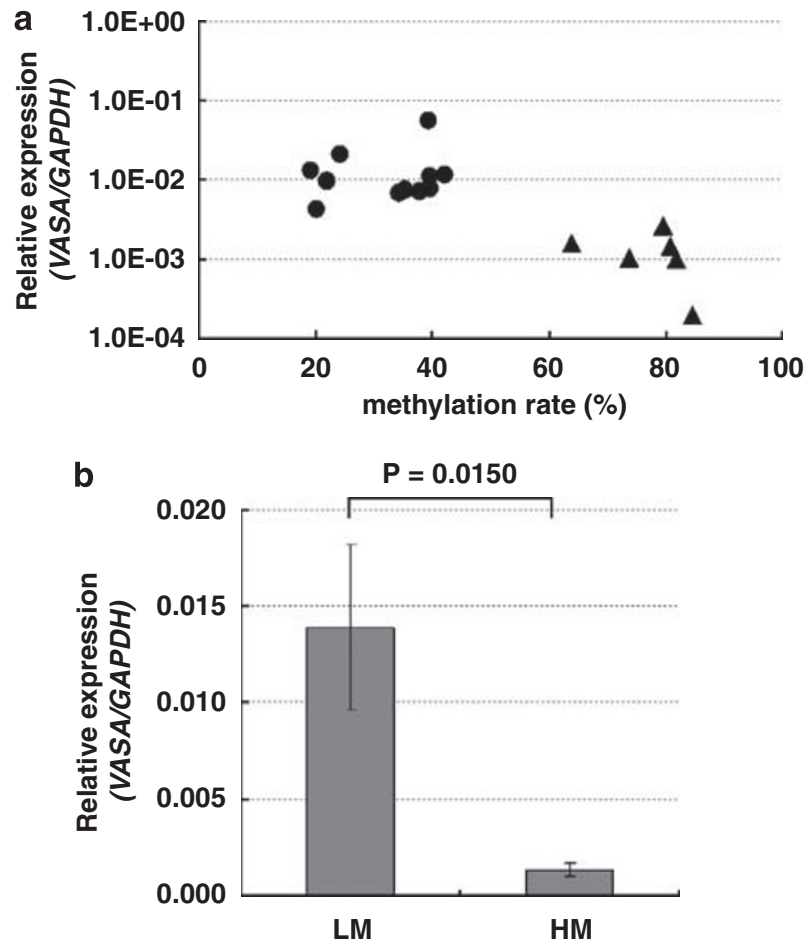

confirmed MA. Despite an apparently high level of VASA TDMR methylation, very few spermatozoa were present in the ejaculate of one patient (MA4). As Mvh-deficient mice are genetically homogeneous, the histology of testis may have a homogeneous appearance. However, the histology in most of the testicular biopsies is clinically heterogeneous. $^{28}$

In general, MA is clinically characterized by an absence of late spermatogenesis, as detected on testicular biopsy. Testicular biopsy appears not to reflect the function of the entire testicular areas, ${ }^{29}$ because the production of spermatozoa occurs in a multifocal (or 'patchy') distribution. ${ }^{30,31}$ Patients with MA have no or very few spermatozoa in their ejaculate. This may also imply that heterogeneity in testicular histology can cause heterogeneity in the DNA methylation status of the VASA gene.

In males, meiosis occurs by two successive divisions. In the first division of meiosis, primary spermatocytes develop into secondary spermatocytes and the second meiotic division yields round

Figure 6 Classification between high and low methylated tissue-specific differentially methylated regions (TDMRs) groups in patients with maturation arrest. (a) Scatter plot of the methylation rate and relative expression of VASA in patients with maturation arrest. Closed circles indicate low methylation of TDMR (LM) group, closed triangles depict high methylation of TDMR (HM) group. (b) The relative expression level of VASA between HM $(n=6)$ and LM group $(n=11)$ in patients with maturation arrest. 
spermatids. The genetic regulation of meiosis during spermatogenesis remains unclear, although some meiotic failure is believed to result from meiotic recombination frequencies ${ }^{32}$ or from the methylation state of the promoter regions of the genes. ${ }^{1,2}$

On the one hand, ejaculate spermatozoa contain a complex of mRNAs and can now be assessed in a noninvasive examination of testis-specific infertility. ${ }^{31}$ It has been reported that the expression of VASA mRNA is significantly decreased in the ejaculate sperm of oligozoospermic patients. ${ }^{33}$ However, the cause of the absence of VASA mRNA expression was not investigated in these reports. The results from this study show that an aberrant methylation of TDMRs may be the underlying cause of meiotic arrest, leading to infertility. This study also may imply heterogeneous testicular expression of the VASA gene. These data raise the possibility that TDMR methylation in human VASA may contribute to some forms of idiopathic male infertility.

In conclusion, we report the novel finding that VASA TDMR methylation is significantly higher among MA patients, in whom the VASA gene expression was silenced. This finding represents an important contribution to the molecular basis of meiotic arrest as one possible cause of idiopathic infertility.

\section{ACKNOWLEDGEMENTS}

This study was supported in part by a Grant-in-Aid for scientific research from the Japanese Ministry of Education, Science, Sports, and Culture (no. 21791496, no.19390412); 'Academic Frontier' Project for Private Universities: matching fund subsidy from MEXT (Ministry of Education, Culture, Sports, Science and Technology), 2006-2010; and Nihon University Research Grant (2006-2007). The authors thank Dr Nobutoshi Kanno for technical assistance.

1 Biermann, K. \& Steger, K. Epigenetics in male germ cells. J. Androl. 28, 466-480 (2007).

2 Allegrucci, C., Thurston, A., Lucas, E. \& Young, L. Epigenetics and the germline. Reproduction 129, 137-149 (2005).

3 Riggs, A. D. X inactivation, differentiation, and DNA methylation. Cytogenet. Cell Genet. 14, 9-25 (1975).

4 Holliday, R. \& Pugh, J. E. DNA modification mechanisms and gene activity during development. Science 187, 226-232 (1975)

5 Matsuyama, T., Kimura, M. T., Koike, K., Abe, T., Nakano, T. \& Asami, T. et al. Global methylation screening in the Arabidopsis thaliana and Mus musculus genome: applications of virtual image restriction landmark genomic scanning (Vi-RLGS). Nucleic Acids Res. 31, 4490-4496 (2003).

6 Song, F., Smith, J. F., Kimura, M. T., Morrow, A. D., Matsuyama, T. \& Nagase, H. et al. Association of tissue-specific differentially methylated regions (TDMs) with differential gene expression. Proc. Natl Acad. Sci. USA 102, 3336-3341 (2005).

7 Cho, J. H., Kimura, H., Minami, T., Ohgane, J., Hattori, N. \& Tanaka, S. et al. DNA methylation regulates placental lactogen I gene expression. Endocrinology 142, 3389-3396 (2001).

8 Hattori, N., Nishino, K., Ko, Y. G., Hattori, N., Ohgane, J. \& Tanaka, S. et al. Epigenetic control of mouse Oct-4 gene expression in embryonic stem cells and trophoblast stem cells. J. Biol. Chem. 279, 17063-17069 (2004).

9 Ching, T. T., Maunakea, A. K., Jun, P., Hong, C., Zardo, G. \& Pinkel, D. et al. Epigenome analyses using BAC microarrays identify evolutionary conservation of tissue-specific methylation of SHANK3. Nat. Genet. 37, 645-651 (2005).
10 Kondo, T., Bobek, M. P., Kuick, R., Lamb, B., Zhu, X. \& Narayan, A. et al. Wholegenome methylation scan in ICF syndrome: hypomethylation of non-satellite DNA repeats D4Z4 and NBL2. Hum. Mol. Genet. 9, 597-604 (2000).

11 Kitamura, E., Igarashi, J., Morohashi, A., Hida, N., Oinuma, T. \& Nemoto, N. et al. Analysis of tissue-specific differentially methylated regions (TDMs) in humans. Genomics 89, 326-337 (2007).

12 Fujiwara, Y., Komiya, T., Kawabata, H., Sato, M., Fujimoto, H. \& Furusawa, M. et al. Isolation of a DEAD-family protein gene that encodes a murine homolog of Drosophila vasa and its specific expression in germ cell lineage. Proc. Natl Acad. Sci. USA 91, 12258-12262 (1994).

13 Noce, T., Okamoto-Ito, S. \& Tsunekawa, N. Vasa homolog genes in mammalian germ cell development. Cell Struct. Funct. 26, 131-136 (2001).

14 Tanaka, S. S., Toyooka, Y., Akasu, R., Katoh-Fukui, Y., Nakahara, Y. \& Suzuki, R. et al. The mouse homolog of Drosophila Vasa is required for the development of male germ cells. Genes Dev. 14, 841-853 (2000).

15 Castrillon, D. H., Quade, B. J., Wang, T. Y., Quigley, C. \& Crum, C. P. The human VASA gene is specifically expressed in the germ cell lineage. Proc. Natl Acad. Sci. USA 97, 9585-9590 (2000).

16 Abdelhaleem, M. RNA helicases: regulators of differentiation. Clin. Biochem. 38, 499-503 (2005).

17 Cordin, O., Banroques, J., Tanner, N. K. \& Linder, P. The DEAD-box protein family of RNA helicases. Gene 367, 17-37 (2006).

18 Rocak, S. \& Linder, P. DEAD-box proteins: the driving forces behind RNA metabolism. Nat. Rev. Mol. Cell Biol. 5, 232-241 (2004).

19 Linder, P. \& Lasko, P. Bent out of shape: RNA unwinding by the DEAD-box helicase Vasa. Cell 125, 219-221 (2006).

20 Schmid, S. R. \& Linder, P. D-E-A-D protein family of putative RNA helicases. Mol. Microbiol. 6, 283-291 (1992).

21 Silverman, E., Edwalds-Gilbert, G. \& Lin, R. J. DExD/H-box proteins and their partners: helping RNA helicases unwind. Gene 312, 1-16 (2003).

22 Megosh, H. B., Cox, D. N., Campbell, C. \& Lin, H. The role of PIWI and the miRNA machinery in Drosophila germline determination. Curr. Biol. 16, 1884-1894 (2006).

23 Fukushima, M., Koh, E., Choi, J., Maeda, Y., Namiki, M. \& Yoshida, A. Reevaluation of azoospermic factor $\mathrm{c}$ microdeletions using sequence-tagged site markers with confirmed physical positions from the GenBank database. Fertil. Steril. 85, 965-971 (2006).

24 Hung, A. J., King, P. \& Schlegel, P. N. Uniform testicular maturation arrest: a unique subset of men with nonobstructive azoospermia. J. Urol. 178, 608-612; discussion 612 (2007).

25 Su, L. M., Palermo, G. D., Goldstein, M., Veeck, L. L., Rosenwaks, Z. \& Schlegel, P. N. Testicular sperm extraction with intracytoplasmic sperm injection for nonobstructive azoospermia: testicular histology can predict success of sperm retrieval. J. Urol. 161, 112-116 (1999).

26 Ezeh, U. I., Moore, H. D. \& Cooke, I. D. Correlation of testicular sperm extraction with morphological, biophysical and endocrine profiles in men with azoospermia due to primary gonadal failure. Hum. Reprod. 13, 3066-3074 (1998).

27 Ehrich, M., Nelson, M. R., Stanssens, P., Zabeau, M., Liloglou, T. \& Xinarianos, G. et al. Quantitative high-throughput analysis of DNA methylation patterns by base-specific cleavage and mass spectrometry. Proc. Natl Acad. Sci. USA 102, 15785-15790 (2005).

28 Dasoula, A., Georgiou, I., Kontogianni, E., Sofikitis, N. \& Syrrou, M. Methylation status of the SNRPN and HUMARA genes in testicular biopsy samples. Fertil. Steril. 87, 805-809 (2007).

29 Ramasamy, R. \& Schlegel, P. N. Microdissection testicular sperm extraction: effect of prior biopsy on success of sperm retrieval. J. Urol. 177, 1447-1449 (2007).

30 Hauser, R., Botchan, A., Amit, A., Ben Yosef, D., Gamzu, R. \& Paz, G. et al. Multiple testicular sampling in non-obstructive azoospermia-is it necessary? Hum. Reprod. 13, 3081-3085 (1998).

31 Ostermeier, G. C., Dix, D. J., Miller, D., Khatri, P. \& Krawetz, S. A. Spermatozoal RNA profiles of normal fertile men. Lancet 360, 772-777 (2002).

32 Gonsalves, J., Sun, F., Schlegel, P. N., Turek, P. J., Hopps, C. V. \& Greene, C. et al. Defective recombination in infertile men. Hum. Mol. Genet. 13, 2875-2883 (2004).

33 Guo, X., Gui, Y. T., Tang, A. F., Lu, L. H., Gao, X. \& Cai, Z. M. Differential expression of VASA gene in ejaculated spermatozoa from normozoospermic men and patients with oligozoospermia. Asian J. Androl. 9, 339-344 (2007). 